\title{
Entwicklung eines Systems zur Auskleidung von Sprengbohrlöchern beim Einsatz von losem Emulsionssprengstoff
}

\author{
Thomas Seidl, Christian Heiss und Maximilian Föger
}

Lehrstuhl für Bergbaukunde, Bergtechnik und Bergwirtschaft, Montanuniversität Leoben, Leoben, Österreich

Eingegangen 12. Februar 2018; angenommen 13. Februar 2018; online publiziert 1. März 2018

\begin{abstract}
Zusammenfassung: Der Einsatz von vor Ort sensibilisiertem Pumpsprengstoff bietet gegenüber patroniertem Sprengstoff Vorteile hinsichtlich Sicherheit, Arbeitsabläufen, Lagerhaltung und Transport. Klüfte und Hohlräume mit Verbindung zum Bohrloch werden allerdings ebenfalls mit Sprengstoff gefüllt, wodurch keine homogene Ladesäule besteht. Der somit unkontrollierte Energieeintrag ins Gebirge erhöht das Risiko von Steinflug und beeinflusst die Erschütterungs- und Lärmemissionen negativ. Durch unvollständige Detonation im Hauwerk verbleibende Sprengstoffreste können Nitratemissionen verursachen.

Im Rahmen des EU Horizon 2020 Projekts "Sustainable Low Impact Mining - SLIM“ wird auf Basis des bestehenden ContBlast-Systems eine Methode entwickelt, um Sprengbohrlöcher mit einem Kunststoffschlauch auszukleiden. Dabei wird eine Einheit, die den zusammengefalteten Kunststoffschlauch enthält, mit dem Ladeschlauch ins Bohrlochtiefste abgelassen und während dem Ladevorgang ausgefaltet und mit Sprengstoff gefüllt. Versuche zeigen die Funktion dieses Systems unter kontrollierten Bedingungen. Beim praktischen Einsatz im Bohrloch ergeben sich Risiken durch nachbrechende Steine, die die Einheit verklemmen können. Zukünftig geplante Versuche zielen auf die Risikominimierung dieses Problems ab.
\end{abstract}

Schlüsselwörter: Bohrlochauskleidung, Pumpsprengstoff, Tagbau, EU Horizon 2020

Design of a System for Lining of Blast Holes While Charging with Bulk Emulsion Explosives

Abstract: Site sensitized emulsion explosives offer several advantages over cartridged explosives regarding safety,

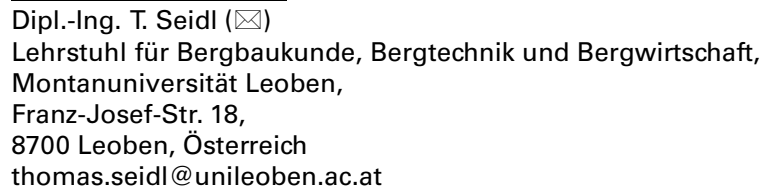

working conditions, storage and transport. Faults and voids connected to the borehole also fill with explosives, creating an inhomogeneous charge column. This increases the risk of flyrock, vibration and noise emissions. Undetonated amounts of explosives remaining in the muck pile may lead to nitrate emissions.

As part of the EU Horizon 2020 project "Sustainable Low Impact Mining - SLIM", a method for lining blast holes while charging them with bulk emulsions based on the ContBlast system should be developed. A unit containing a folded plastic hose is attached to the charging hose and lowered to the bottom of the hole. While emulsion explosives are pumped in, the hose unfolds and the unit is pushed up towards the top of the hole.

Tests in a defined environment proved the principal function of the system. When testing the system in an open pit mine, problems involving the container getting jammed in the drillhole arose on occasions. Future tests therefore focus on practical handling of the system and dealing with the risk of getting jammed.

Keywords: Blast hole lining, Site sensitized emulsion explosives, Open pit mining, EU Horizon 2020

\section{Einleitung}

Der Einsatz von losem, vor Ort sensibilisiertem Emulsionssprengstoff bietet wesentliche Vorteile gegenüber patroniertem Sprengstoff hinsichtlich Arbeitssicherheit, Transport sowie Lagerhaltung, da der Sprengstoff erst durch die Sensibilisierung im Bohrloch zündfähig wird und bis zu diesem Zeitpunkt nur mit Einzelkomponenten hantiert werden muss. Nachteile ergeben sich allerdings im klüftigen und wasserführenden Gebirge. Durch die pastöse, fließfähige Konsistenz dringt der Sprengstoff in Klüfte und Hohlräume, wodurch sich in diesen Bereichen erhöhte Sprengstoffmengen ergeben. Durch die somit partiell erhöhte Energiekonzentration besteht das Risiko von 
Steinflug, ein erhöhter Anteil von Feingut im Hauwerk sowie erhöhter Sprengstoffverbrauch. In engen Klüften und Hohlräumen ist der kritische Durchmesser der Ladesäule nicht mehr gegeben, wodurch die vollständige Umsetzung des Sprengstoffs nicht gewährleistet werden kann und Reste im Hauwerk verbleiben. Dies kann zu Nitratemissionen im Bergbau, auf der Halde oder am Einsatzort des Rohstoffs führen sowie den Aufbereitungsprozess beeinflussen.

Im Rahmen des EU Horizon 2020 Projekts "Sustainable Low Impact Mining" (SLIM) forscht der Lehrstuhl für Bergbaukunde an der Montanuniversität Leoben gemeinsam mit einem Konsortium von europäischen Partnerinstitutionen und Firmen an effizienten und nachhaltigen Sprengtechniken zur Gewinnung von kleinen und komplexen Rohstofflagerstätten. Um die oben genannten Probleme zu lösen, wird als Teil des Arbeitspakets 3 "Umwelteinflüsse und Sicherheit" ein Prototyp zur Auskleidung von Sprengbohrlöchern und damit zur Reduktion von Nitrat-Emissionen und besseren Kontrolle der Ladesäule entwickelt [1,2].

Es ergeben sich zwei Hauptanforderungen an den zu entwickelnden Prototypen: Durch Einbringen einer Bohrlochauskleidung in Form eines Kunststoffschlauches wird eine durchgängige, zylindrisch geformte Ladesäule sichergestellt und diese Ladesäule gegen anstehendes oder vorbeiströmendes Gebirgswasser geschützt. Die Auskleidung erfolgt dabei während dem Einbringen des Sprengstoffs ( „lining while charging“), da der klassische Einsatz von auf Rollen gelieferten Kunststoffschläuchen, die vor dem Sprengstoff ins Bohrloch eingebracht werden müssen, sehr arbeitsaufwändig ist.

\section{Bohrlochauskleidung}

Zum Einsatz von Kunststoffschläuchen und losen Emulsionssprengstoffen konnte auf Erfahrungen aus dem früheren Projekt ContBlast zurückgegriffen werden, welches im SLIM-Projekt explizit als Entwicklungsgrundlage vorgesehen ist. Dieses System ermöglicht die Herstellung von entkoppelten Ladesäulen zum schonenden Sprengen (Kontursprengen). Dabei wird der Sprengstoff während des Ladevorgangs in einen Kunststoffschlauch eingebracht, dessen Durchmesser kleiner ist als der Bohrlochdurchmesser. Der Schlauch ist dabei in einem Schutzgehäuse - der ContBlastEinheit, die auch Zünder und Booster aufnimmt - verstaut und wird während dem Ladevorgang vom Bohrlochtiefsten aus entfaltet. Um eine optimale Entkopplung vom Gebirge und einen gleichmäßigen Ringraum zu gewährleisten, wird der gefüllte Kunststoffschlauch in regelmäßigen Abständen durch Ringe im Bohrloch zentriert. Dieses System konnte in bis zu vier Meter langen horizontalen Bohrlöchern für den Streckenvortrieb im untertägigen Bergbau bereits erfolgreich erprobt werden und wird für eine Anwendung im Tagebau bei voller Ausladung des Bohrloches mit Sprengstoff adaptiert $[3,4]$.

Folgende Arbeitsschritte sind zum Auskleiden und Besetzen eines Bohrlochs erforderlich:

- Anbringen der vorgefertigten Einheit am Ladeschlauch

- Montage von Booster und Zünder an der Einheit
- Einbringen der Einheit mit dem Ladeschlauch bis in Bohrlochtiefste

- Einpumpen des Emulsionssprengstoffs, dabei wird der Kunststoffschlauch ausgefaltet und der Ladeschlauch mit der Einheit durch den Sprengstoff nach oben gedrückt.

- Die konstruktiven Elemente der Einheit werden nach Ende des Pumpvorgangs mit dem Ladeschlauch aus dem Bohrloch gezogen, lediglich der Kunststoffschlauch verbleibt im Bohrloch.

- Redundanter Zünder und Booster werden innerhalb des Kunststoffschlauches im Bohrloch platziert.

\section{Prototypentwicklung}

Entsprechend den Vorgaben im SLIM Projekt wurde der Prototyp nach folgenden Spezifikationen entwickelt:

- 15-20 m Bohrlochlänge (bis zu 30 m konnten für Versuche realisiert werden)

- $89 \mathrm{~mm}$ Bohrlochdurchmesser

- $90 \mathrm{~mm}$ Durchmesser Auskleidungsschlauch

- Emulsions-Ladeschlauchdurchmesser kleiner $30 \mathrm{~mm}$

- Mischladeeinheit mit Statikmischer am Ende des Ladeschlauchs

- Anbringen von Zünder und Booster vor Ort

Wesentlicher Unterschied zum ContBlast-System ist, dass durch das gewünschte vollständige Ausladen des Bohrloches der Kunststoffschlauch einen größeren Durchmesser als das Schutzrohr aufweist und somit der Ausfaltmechanismus neu konzipiert werden musste sowie die Zentrierringe entfallen. Die Herausforderung liegt darin, das gleichmäßige, einlagige Ausfalten des Schlauches zu gewährleisten, ohne einen zu großen Widerstand zu verursachen. Die vorgesehenen Bohrlochabmessungen erfordern eine wesentlich größere und längere Bauform, um die notwendige Menge Kunststoffschlauch aufzunehmen. Dies bedingt auch eine Änderung der Sprengstoffzuführung, da der Sprengstoff nach Austritt aus dem Ladeschlauch (wo durch den Statikmischer die Emulsionsmatrix und das zusätzlich als Schmierfilm dienende Reaktiv vermischt werden) nur mehr schwer pumpbar ist. Während das ContBlast-System mit einer Läge von ca. $50 \mathrm{~cm}$ noch auf den Ladeschlauch aufgesteckt werden kann, muss bei nun erforderlichen Baugrößen von bis zu $190 \mathrm{~cm}$ (für $30 \mathrm{~m}$ Bohrlöcher) der Ladeschlauch durch die Einheit ins Bohrlochtiefste geführt werden. Aus Kosten- und Fertigungsgründen wurde auf eine möglichst einfache Konstruktion ohne bewegliche Bauteile Wert gelegt.

Eine Einheit besteht aus dem zwischen Zentralrohr und Schutzrohr gefaltet verstauten Kunststoffschlauch sowie dem Ausfaltmechanismus am unteren Ende. Durch den modularen Aufbau ist das Anpassen der Schlauchlänge an unterschiedliche Bohrlochlängen problemlos möglich. Die Anpassung an einen geringfügig größeren Bohrlochdurchmesser erscheint machbar, die Funktionsfähigkeit muss allerdings in weiteren Tests nachgewiesen werden. Weiterreichende Änderungen erfordern eine Neukonstruktion. 


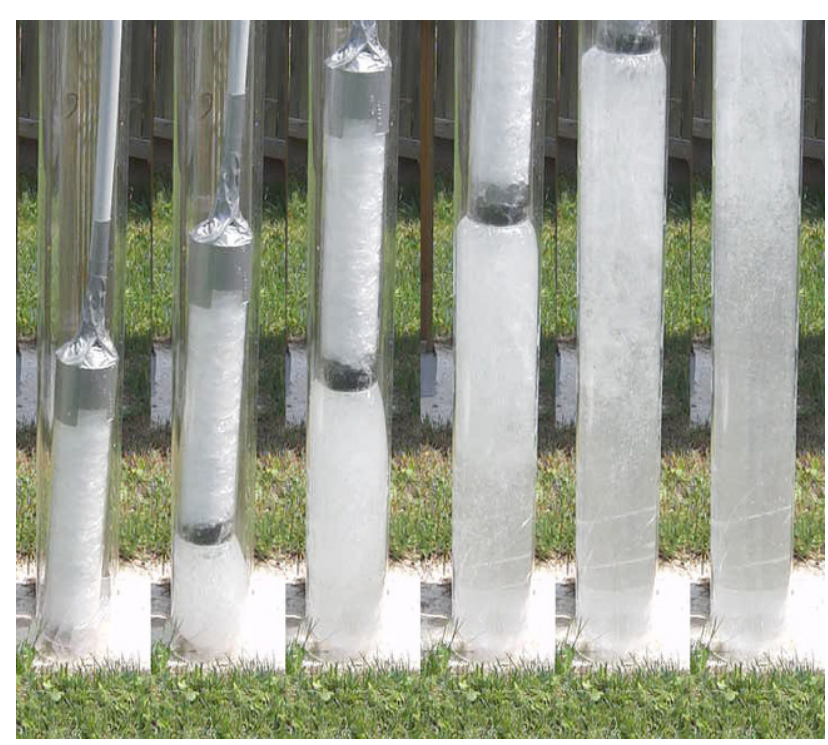

Abb. 1: Versuch eines Befüllvorgangs mit Wasser im transparenten Kunststoffrohr

Bei der Entwicklung wurde aus Kosten- und Effizienzgründen weitgehend auf handelsübliche Komponenten zurückgegriffen. Lediglich die Ausfaltvorrichtung wurde als spezifisch konstruiertes Bauteil mittels 3D-Druck hergestellt. Bei Serienfertigung ist der Einsatz eines Drehoder Spritzgussteiles vorgesehen. Die Schutzrohre sind derzeit aus transparentem Kunststoff gefertigt, um das Faltverhalten des Kunststoffschlauches in den Versuchen zu beurteilen. In weiterer Folge sollen dafür versiegelte Kartonrohre zum Einsatz kommen.

\subsection{Versuche unter Laborbedingungen}

Um die ordnungsgemäße Funktion, speziell das planmäßige Ausfalten des Kunststoffschlauches zu testen, wurden erste Tests mit Wasser durchgeführt und das Bohrloch durch ein transparentes Kunststoffrohr simuliert.

Abb. 1 zeigt, von links nach rechts, den zeitlichen Ablauf eines Befüllvorganges mit Wasser im transparenten Kunststoffrohr. Am linken Bild befindet sich ein $40 \mathrm{~cm}$ langes Modul vor Beginn des Befüllens am tiefsten Punkt des simulierten Bohrlochs. Während dem Befüllvorgang wird der Kunststoffschlauch aus der Einheit entfaltet und legt sich an die Bohrlochwand. Zeitgleich wird die Einheit nach oben gedrückt.

Um die Tauglichkeit des Systems zum Überbrücken von Klüften zu simulieren, wurde zwischen zwei Rohrsegmenten ein entsprechender Abstand frei gehalten und der Kunststoffschlauch mit Wasser eingebracht. Dabei zeigt sich, dass der derzeit verwendete Kunststoffschlauch mit einer Stärke von $100 \mu \mathrm{m}$ dem im Bohrlochtiefsten erwarteten Druck von 2 bar über eine freie Strecke von ca. $10 \mathrm{~cm}$ widerstehen kann und sich lediglich aufwölbt. Darüber hinaus kann es zum schlitzförmigen Platzen des Schlauches kommen.
Die letzten Versuche zum Füllen mit Pumpsprengstoff verliefen zufriedenstellend, nachdem, basierend auf den ersten Versuchen, die Konstruktion in geeigneter Weise adaptiert worden ist.

Bei allen bisherigen Versuchen im Bergbau standen nur wassergefüllte Bohrlöcher zur Verfügung. Bei ersten Tests zum Ein- und Ausbringen der Einheit im Bohrloch zeigt sich, dass der Auftrieb des Kunststoffkörpers zu groß ist, um durch das Eigengewicht des gefüllten Ladeschlauches unter Wasser gedrückt zu werden. Hier muss noch eine passende Lösung gefunden werden. Im trockenen Teil der Bohrlöcher konnte die Einheit gut bewegt werden, allerdings besteht durch den verhältnismäßig kleinen Ringspalt von nur $10 \mathrm{~mm}$ zwischen Einheit und Bohrlochwand die Gefahr, dass sich die Einheit verkantet oder durch nachbrechende Steine verklemmt wird. Aus Sicherheitsgründen wurden die Prototypen daher noch nicht mit Sprengstoff im Bohrloch getestet, diese Versuche sind für Frühjahr 2018 geplant.

\section{Schlussfolgerungen und Ausblick}

Die bisherigen Versuche haben gezeigt, dass das Ausfalten des Kunststoffschlauches und das Auskleiden des Bohrloches unter kontrollierten Bedingungen funktionieren. Beim Einsatz im Bohrloch bestehen allerdings Probleme durch nachbrechende Steine und das Risiko, dass die Einheit nicht mehr aus dem Bohrloch entfernt werden kann bzw. das Bohrloch nicht vollständig geladen ist. Der Fokus für die weiteren noch geplanten Versuche liegt daher auf der praktischen Handhabung des Systems und entsprechenden Vorbeugemaßnahmen, um das Steckenbleiben der Einheit im Bohrloch zu verhindern.

Danksagung. Die präsentierten Arbeiten werden durch die EU Horizon 2020 Forschungs- und Innovationsprogramme unter Grant Agreement No 730294 ("Sustainable Low Impact Mining solution for exploitation of small mineral deposits based on advanced rock blasting and environmental technologies", SLIM) gefördert.

Funding. Open access funding provided by Montanuniversity Leoben.

Open Access Dieser Artikel wird unter der Creative Commons Namensnennung 4.0 International Lizenz (http://creativecommons.org/licenses/ by/4.0/deed.de) veröffentlicht, welche die Nutzung, Vervielfältigung, Bearbeitung, Verbreitung und Wiedergabe in jeglichem Medium und Format erlaubt, sofern Sie den/die ursprünglichen Autor(en) und die Quelle ordnungsgemäß nennen, einen Linkzur Creative Commons Lizenz beifügen und angeben, ob Änderungen vorgenommen wurden.

\section{Literatur}

1. Sifferlinger, N. A.; Ouchterlony, F; Brechelmacher, A.: Das europäische Horizon 2020-Forschungsprojekt "Sustainable Low Impact Mining - SLIM", BHM Berg- und Hüttenmännische Monatshefte, 163 (2018), H. 2, S. 52-56 (DOI: https://doi.org/10.1007/s00501-018-07014)

2. Grant Agreement Number 730294 - SLIM, European Commission, Executive Agency for Small and Medium-sized Enterprises (EASME), 730294-SLIM-H2020-SC5-2016-2017/H2020-SC5-2016-OneStageB

3. Ivanova, R.; Ganster, M.; Moser, P.; Bauer, F: Development of a new contour blasting system using bulk emulsions, in: Proceedings of the ISEE annual conference on Explosives \& Blasting Technique, 
Nashville, USA, 2012. Publ. by: International Society of Explosives

Engineers (ISEE), Cleveland, Ohio, USA, 2012, pp 253-263

4. Moser, P.; Ouchterlony, F.: A device, charging unit and method of filling a borehole with an explosive material, EP/07010995.4, University of Leoben, Austria, 2008 